\title{
Understanding and Proposing a Design Rationale of Digital Games based on Brain-Computer Interface: Results of the AdmiralMind Battleship Study
}

\author{
Alessandro Luiz Stamatto Ferreira, Juvane Nunes Marciano, \\ Leonardo Cunha de Miranda, Erica Esteves Cunha de Miranda \\ Department of Informatics and Applied Mathematics \\ Federal University of Rio Grande do Norte (UFRN) \\ Natal, RN, Brazil \\ alexmatto@ppgsc.ufrn.br, juvane@ppgsc.ufrn.br, leonardo@dimap.ufrn.br, erica@dimap.ufrn.br
}

\begin{abstract}
Brain-Computer Interfaces (BCIs) present great potential to the field of digital games, though the design of this kind of interface is still a challenge for most of the game designers and developers. In this work it is presented a literature review about digital games based on BCI, aiming to analyze the interaction design of these games, to identify the approaches applied, limitations and implications related to BCIs design. After the review, we accomplish a reflection about the design decisions involved in the development of digital games based on BCI. This approach leads to the construction of a design rationale developed to support the process of $\mathrm{BCI}$-based games, with its use established on the design of a battleship game based on BCI. As contributions of this work, we highlight the literature review, design rationale and results of the study accomplished.
\end{abstract}

Keywords-BCI, EEG, P300, SSVEP, HCI, designer.

\section{INTRODUCTION}

The digital games industry is growing increasingly, attracting all kinds of people with innovations in several areas, such as hardware, software, 3D graphical interfaces and new ways of interaction. The scope of new interaction ways has motion sensors - e.g. Nintendo WiiMote sensors accelerometers in smartphones, visual recognition of gestures e.g. through Microsoft Kinect - and augmented reality. Even in this scope, one of the most promising interaction ways for the "future" is the one provided by Brain-Computer Interfaces (BCIs), which allows interaction desired by many users/players, i.e. control of games only through the brain, without using any physical artifact of interaction, even the innovative ones as the Adjustable Interactive Rings [35][36].

Nowadays, electroencephalography devices (EEG) are able to provide a $\mathrm{BCI}$ where the interaction is done by capturing the user's brain activity. The development of commercial ${ }^{1}$ EEG headsets $^{2}$, for example, by NeuroSky (e.g., ThinkGear AM, MindWare, Mindwave Mobile and MindSet), by Emotiv (e.g., EPOC Neuroheadset and EEG Neuroheadset) and by BCINet (e.g., NIA Game Controller), allows end users to submerse in

\footnotetext{
${ }^{1}$ Information about each mentioned headset can be found, respectively, in: http://www.neurosky.com, http://www.emotiv.com, and http://www.bcinet.com

${ }^{2}$ The term "headset" is used in this work to unify the various formats of capture devices that may include headbands, caps, bandanas, headphones and helmets.
}

this "new" interaction paradigm, also in the context of digital games. The three most used detection techniques in BCI-based systems are: (i) neurofeedback, in which Alpha and Beta waves are used to estimate focus, relaxing/meditation and concentration of the user; (ii) visual stimuli, in which an element being watched by the user is recognized by detecting brain response to a visual effect that acts as stimulus; the detection depends on the visual stimulus used. A transient stimulation is characterized by elements that blink one by one and when the target element lights, that one observed by the user, the brain answers with a "surprise" wave - the P300 detected by an EEG device. In an oscillating stimulus - called Steady State Visually Evoked Potential (SSVEP) - all visual elements blink simultaneously, each at a different frequency, which causes the user's brain to respond in a frequency similar to the frequency of oscillation of the target element; and (iii) imagined movement, in which it is possible to detect kinetic thoughts as, for example, the imagination of the user's right hand opening and closing, thanks to the synchronization and desynchronization of the Mu rhythm ${ }^{3}$.

For this work, we start from previous results already achieved with accomplishment of research in the field of BCI. In [18], we describe the state of the art of interactive systems based on BCI, as well as present and discuss the main challenges of this domain. In the present work, our focus is to explore, specifically, digital games based on BCI, therefore, we investigate $\mathrm{BCI}$ in the digital games scenario, in order to identify approaches, its limitations, and implications related to the design of BCIs. Also, we develop a design rationale ${ }^{4}$, which supports the design process of BCI-based games. Still, the developed design rationale, was applied in the design process of a battleship game explaining and discussing, in this study, the key issues in designing a BCI game.

\footnotetext{
${ }^{3} \mathrm{Mu}$ rhythm is a pattern of brain electrical activity that occurs in the motor cortex, strongly related to the control of voluntary movements. A person suppresses this pattern when performing, imagining, or observing a motor action.

${ }^{4}$ Design rationale [27] is a document with an explicit listing of decisions involved in the design process. Each decision must contain the possible alternatives, their reasons and pro and con arguments.
} 
This paper is organized as follows: Section II describe the related works; Section III presents a literature review about BCI-based games; Section IV describes the developed design rationale; Section IV presents and discusses the results of the application of the design rationale in the design process of a BCI-based game; and Section VI concludes the paper.

\section{RELATED WORKS}

Lotte [10] identifies BCI challenges in the context of games, highlighting five drawbacks of current BCIs: (i) the user needs to be in a stationary position, since movements can cause interference in the captured signal; (ii) boredom for the user to calibrate the BCI; (iii) discomfort in the use of headsets, due to the their limitations, as the need to use gel in the sensors; (iv) high error rate; and (v) limited set of mental activities that can be detected with good accuracy. On the other hand, this author defends the potential of BCIs, explicating, as an example, the use of game's difficulty setting based on the user's tension. Gürkök et al. [11] argue that BCI games are guided by two principles: motivation ("why" play), and interaction paradigm ("how" to play). These authors then identify three elements that can motivate players, i.e. challenge, fantasy, and social. The authors also define three interaction paradigms, i.e. concentration, imagined movement, and response to stimuli, and finally classify four BCI games according to each of these elements of motivation and interaction paradigms.

Sung et al. [15] created an architecture for the development of serious games, i.e., games where the entertainment is not the main goal, but factors such as education, training or simulation. The process proposed by these authors aims to separate those involved on the development, in specific and specialized roles where BCI experts create templates that can be used by those who have no knowledge in the field, as game experts. These authors also show creation tools, based on templates, for game development. Kaplan et al. [17] accomplish a literature review about BCI games that use, specifically, the P300 approach, identifying negative factors for user experience, and how these factors have been overcome.

Bos et al. [26] present the factors and challenges of HumanComputer Interaction (HCI) involved in BCI games, with a study focused on usability and user experience. These authors conclude that more research is needed in real usage scenarios with users acting naturally, and the HCI community involvement is crucial to the improvement of this interaction way. Marshall et al. [30] present a review of BCI games, classifying them into conventional genres. These authors discuss the current use of BCIs in every game genre and based on that, make recommendations for the best use of BCIs for each of the genres presented. On the other hand, van Veen [19] evaluates the current BCI games under the criterion of the paradox of control, which, according to the author, is a requirement that makes an interactive system be considered a game.

Unlike the works mentioned above (related), this work focuses on the interaction involved in games that use this "new" way of interaction with digital games, regardless of the $\mathrm{BCI}$ detection approach, and also presents a design rationale that supports game designers with the issues involved in the design of BCI games.

\section{LITERATURE REVIEW OF GAMES BASED ON BCI}

Initially, we present a literature review of BCI games, as a key part of this work, since through this review we will better understand the impact of this interaction way in the design of digital games. Thus it is important to highlight that our intention with this section is not to present an exhaustive list of digital games based on BCI, but a selection of the main works that contribute to a better understanding of design of BCI games. The search strategy consisted of manual and automatic searches in scientific libraries and bibliographic databases. Automatic/manual searches were conducted in IEEE Xplore, ACM DL, Springer, Elsevier, Scielo, Scopus, ISI Web of Knowledge, and Google Scholar. In the search process, it was used a combination of the following keywords (listed below in alphabetical order): "BCI", "brain", "computer", "design", "EEG", "EPOC", "Emotiv", "game", "HCI", "interaction", "interface", "MindSet", "MindWave", "NeuroSky", "P300", "rationale", and "SSVEP". For this study we only selected published papers that, in fact, present a BCI game.

The use of BCI in digital games can be divided into four distinct groups depending on the detection method applied in the BCI game, i.e. (i) neurofeedback based games, such as the measurement of relaxation and/or concentration of the user, (ii) games based in response visual stimuli, (iii) games based on imagined movement, and (iv) games based on hybrid detection, i.e. using a combination of two or more forms as described above in (i) (ii) and (iii). In addition, the game control is considered multimodal when using multiple interaction ways, especially in the input, for example, the use of keyboard and mouse together with a BCI. Therefore, the hybrid detection is related to multiple $\mathrm{BCI}$ detection approaches used together, while multimodal indicates that $\mathrm{BCI}$ and non-BCI interaction ways are used together.

\section{A. BCI Games based on Neurofeedback}

In the Finding Star game, developed by Ko et al. [9], control is multimodal, since the concentration and relaxation of the user are captured by MindSet and the movement of the character is done by a conventional keyboard, and the target by a conventional mouse. The accuracy depends on the user's concentration, i.e. in low concentration, the target becomes unstable; as the former grows, the accuracy of the latter improves. In these battles the character loses energy and need to sleep to recover it. For the game heroine to sleep, it is needed that the user reaches a certain level of relaxing. The user state also affects the visibility of certain items of the game GUI, since some elements appear only when the user is quite concentrated, while others only appear when the user is relaxed.

Kang et al. [48] begin the design of a horror puzzle game that will use a BCI during the stages' design. In the game, the player needs to win riddles/puzzles while horror elements appear (like spirits appearing suddenly). Through a BCI, the player's fear will be evaluated, and through that neurofeedback, it will be possible to maximize the terror of the 
game. In an initial prototyping, the authors have managed to capture the fear of people who watch a horror movie, finding which scenes do cause the greatest fear. This demonstrates that not only concentration or relaxation, fear can be exploited in games with BCI - giving the game the ability to interact directly with the player's feelings, in this case, fear.

Liao et al. [4] developed a more convenient headset, where sensors are placed in a polymer sponge in order to let the user more comfortable. To test the solution, the authors developed Archery Game, a game based on the level of user focus, in which the user should focus on a target for 10 seconds, and then an arrow is fired towards the target. The arrow precision is based on the focus level reached by the user. In an experiment, they compare the performance of a user in a controlled and silent environment, with the performance of the same user in an environment with several distractions, sounds and videos in the sight of the user. There was a decrease in performance that demonstrates the difficulty of users to focus in an uncontrolled environment. Jiang et al. [13] developed a game for rehabilitation, where control is performed by a BCI based on concentration. In the game, the user must maintain a level of attention, to allow a virtual hand to reach and grab a tiny red fruit on a plate. The game aims to assist in the rehabilitation of children with attention deficit.

Coulton et al. [7] created a game for smatphone called Brain Maze that uses the MindSet. The objective of the game is to take a ball from a starting point to an ending point. To move the ball, the smartphone's accelerometer is used. However, some roads are blocked by gates. When the user concentrates in attention, attention gates are open, and if the user relaxes, meditation gates are open. Basori [33] developed the Emotion Walking, a game that uses a BCI headset together with a motion capture glove to detect the user's feelings, such as happiness and anger, and then change the facial expression of an avatar. The walking speed of the avatar depends on the user's level of exciting. The glove tracks the current state of the hand, and improves the accuracy of the feelings captured. According to the authors, if the user is in a fist and tense, then, the user is angrier, while an open hand and relaxed indicates greater peace or happiness.

Bernays et al. [37] developed a 3D maze game in first person, called Lost in The Dark, with a BCI based on neurofeedback. The motion controls are conventional, while neurofeedback acts on various parts of the game. In the game, you must escape a cylindrical maze where a ball - called Light Bot - accompanies the player and lights the way. As the player gets tense or excited, the Light Bot fades, and as the player is calm the light increases. Through the maze, there are locked doors that only open if the player reaches a certain "state of mind", i.e. calm, excited, or tense. The music of the game adapts to the user's state; when the player is calm, music is quiet low and calm, when excited, music is high and fast.

In the game Mind Garden (Fig. 1a), developed by Su et al. [39], the goal is to create and maintain a garden. For this, the player plant trees, and for them to grow, the user must achieve a high level of relaxation. To adorn the garden, the player can also control elements like clouds, sun and rainbow. For these elements to develop, the player needs to achieve a high level of attention, thus, the player must carefully control the attention and relaxation in order to create the desired garden. Yoh et al. [38] add interactivity to the fairy tale "Hansel and Gretel" in the form of a BCI game called NeuroWander (Fig. 1b). In the game you need to reach a certain state of mind at every stage, for the characters in the story to have success. For example, it is necessary to relax to spread bread crumbs, and maintain a high level of attention to successfully push the witch - the villain - into the cauldron.

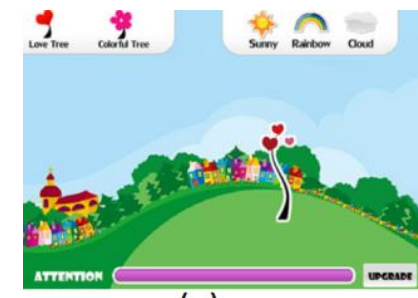

(a)

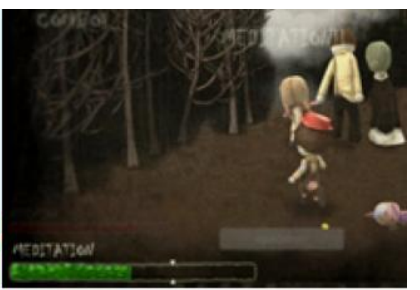

(b)
Fig. 1. Graphical interfaces of BCI games based on neurofeedback (a) Mind Garden (b) NeuroWander. Sources: [39][38].

\section{B. BCI Games based on Visual Stimulus}

Hakvoort et al. [8] developed the Mind the Sheep game (Fig. 2a), whose goal is to control dogs via BCI, to take sheep into a fence. The control uses a multimodal approach, where the visual focus is used to select which dog to move, while the mouse position indicates where the dog must go. The visual focus is detected by a BCI based on SSVEP. On the other hand, Obbink et al. [22] expanded the game cited above - Mind the Sheep - to a BCI cooperative version, where the map/scenario is higher and users should control the dogs together. Some difficulties were encountered, such as the fact that participants avoided talking or gesturing to one another, because it caused interference in the BCI SSVEP detection; this limitation made it difficult to communicate. Another problem identified was the breaking of focus in certain natural situations, such as laughter of a user making the others to do wrong moves. Lalor et al. [12] developed the MindBalance game (Fig. 2b), a game where a character, similar to a frog, must balance up and cross a tightrope. The game is controlled with a BCI based on SSVEP, where the visual stimulus is two flags, increased by the fact that each flag has squares arranged opposite to each other.

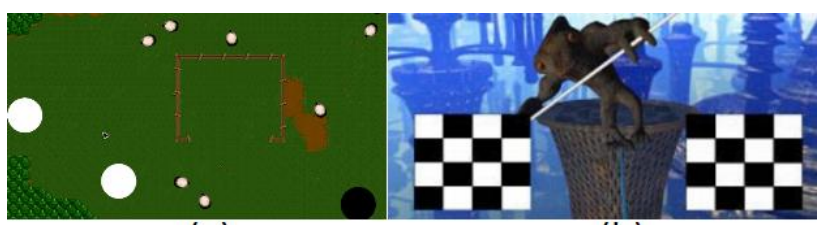

(a)

(b)

Fig. 2. Graphical interfaces of BCI games based on visual stimulus (a) Mind the Sheep (b) MindBalance. Sources: [8][12].

Another game that uses the same scheme of flags is the 2D racing game developed by Martinez et al. [44], where a car must complete laps on a race track; the flags oscillate in SSVEP style. The car is surrounded by four flags, which represent an up/down and left/right move, and the user must 
focus the attention on the flag that represents the direction desired for the car to move.

Vliet et al. [6] developed a game controlled by the EPOC, and compared the performance of this commercial EEG headset destined to the end user, with the high cost headset named actiCAP. To put the headset, it was observed that the EPOC was easier, with the user being able to put it in three minutes, while for put actiCAP it was always necessary someone to assist the user, with an average time of 10 minutes. The game developed is tower defense genre, in which the player must protect a tower from waves of enemies, that appear distant from the tower and go towards it. When an enemy reaches the tower, the user loses the game. The game works in two steps, first the user decides the layout, position, and type of the buildings, and builds the tower defense as preferred. In the second step, the user has no control over the game and the enemies begin to appear, as the user only observes whether the defense architecture will be able to protect the tower of three waves of enemies. The game BCI uses the SSVEP approach, but with only a single flash each time. The performance between EPOC and actiCAP vary depending on the capturing time interval; if it is 0.5 seconds, the EPOC has an accuracy of $66 \%$ versus $79 \%$ of actiCAP, while in the case of 2.2 seconds, EPOC has an accuracy of $85 \%$ versus $88 \%$ of actiCAP.

Chumerin et al. [23] present a maze game (Fig. 3a), where a BCI based on SSVEP is responsible for controlling the character represented by the face of Bart Simpson (from the Simpsons cartoon), which must reach a donut. Four yellow arrows act as visual stimuli. One difficulty in the design of the game is the fact that it takes certain time to capture and classify the EEG signals, which causes a notable delay in the response of the game. To minimize this problem, "hiding" the "delay", the character constantly moves while the command is referring to the next decision point - crossroads - of the maze. On the other side, Maby et al. [20] developed a BCI version of the game Connect Four (Fig. 3b), whose goal is to form a row, column, or diagonal of four coins. Two players, represented by the colors red and yellow, alternate in choosing a column to enter the coin. A P300 visual stimulus is used, in the form of a rectangle of the player's color, flashing on each column, one at a time. Fig. $3 b$ shows a game screen where the visual stimulus is on the penultimate column. The rationale time mitigates the time for choosing a column, giving impression that the game follows the same speed than the conventional version.

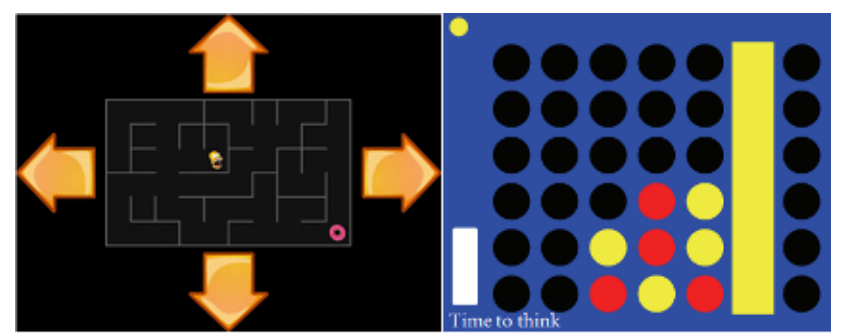

(a)

(b)

Fig. 3. Another graphical interfaces of BCI games based on visual stimulus (a) The Maze (b) Connect Four. Sources: [23][20].
Congedo et al. [1] developed, supported by the Open ViBE ${ }^{5}$ platform, a game with a P300 based BCI, called Brain Invaders, inspired by the classic game Space Invaders. In this game, gray aliens are presented in a grid and one of them is special, being red instead of gray. The aliens flash randomly, the red flashes to blue, while the other flash to a lighter gray; and to destroy an alien, the user must focus on it visually. The user gains after destroying the alien red, or any 14 aliens. The authors state in their game, three enhancements of conventional BCIs: (i) the alien blink in random groups, providing a higher data speed over BCIs which flash one element at a time, and maintaining a good precision; (ii) the time between two flashes is random, reducing predictability, increasing BCI accuracy and game fun; and (iii) increasing the size of target elements during a flash also increases the accuracy, i.e. the red alien's size is increased by $30 \%$ in flashing time.

Angeloni et al. [5] developed a P300 based BCI for a memory game. Five undergraduate students participated in tests with the BCI. Initially each one of them typed words using a BCI speller, aiming to calibrate and configure system parameters. Then they played a game where it is presented a $3 \times 4$ matrix of letters. The authors believe that the memory game can be a good way to train users in BCIs. Another P300 based game is MindGame, by Finke et al. [43], in which the player, represented by a cartoon character, must move by a grid map. This map contains several trees, and the goal of the game is to visit all the trees. Although the grid contain numerous positions, only those in front of trees are explored, i.e. only those that flash - in P300 style - for the player to select one.

\section{BCI Games based on Imagined Movement}

Scherer et al. [2][3] created a BCI called Graz BCI Game Controller, which allows the use of two headsets, the Graz BCI [2] and the EPOC. The BCI was tested in the World of Warcraft (WoW) game, that was not originally designed to operate via BCI. The BCI is based on three imagined movements, the right hand turns the screen to the right, the left hand rotates the screen to the left, and the feet moves the character forward. Additionally, certain actions are triggered automatically when the character is standing in the range required to achieve it. Non-critical actions, like picking up a resource, are activated immediately, while critical actions, such as starting a fight with a monster, are carried out only after a period of time, so that the user can cancel the action by moving the character; the biggest limitation found was the low speed of the BCI. This problem has been soften by the macro system of the game, in which a single command may be interpreted as different actions, depending on the current context, making them more flexible and providing a larger input rate. With the EPOC - Instead of Graz BCI - it is possible to send emoticons based on user's facial expression. In this BCI, user's eyes closed make the character sleep, while a user's smile makes the character laugh.

Leeb et al. [16] developed the game Thinking Penguin to work via BCI, based on the conventional game PlanetPenguin Racer. In the original game - PlanetPenguin Racer - the player should control a penguin down a snowy mountain, collecting

\footnotetext{
${ }^{5}$ http://openvibe.inria.fr.
} 
fish on the way. For the BCI version, the authors modified the game putting all the fish in the air. In this new way, the player must enable jumps through the imagination of the movement to lift both feet - dorsiflexion - to collect the fish. For greater immersion, the game takes place in a virtual reality CAVE system, in which the user is surrounded by walls with projectors targeted to each of them. Thus, the game interface is multimodal with movement of the penguin controlled through a joystick and the jumps through BCI. An experiment showed that the concomitant use of the joystick did not compromise the performance of the BCI. According to the authors, the game requires a short training period.

Badia et al. [47] created a serious game for rehabilitation of people, especially adults, who lost the movement of the arms due to a stroke. The game is in first person and the goal of the game is to "block" balls coming towards the player. The game interface changes its input depending on the severity of motor loss of the player, if the player is able to move the arms, interaction is for computer vision: a camera above the monitor tracks the movement of the arms, while a BCI based on imagined movement is used if the player does not have enough arm motor control. The imagination of arm movement stimulates the brain to regain lost motor skills. The control is done by the imagination of the movement of the right and left arms, beginning at the center of the screen (in front of character's torso) and imagination of one of the arms moves it in the right direction (right arm) or left (left arm). The extent of movement is proportional to the time the player imagine that motion, if it passes a long time imagining, the arm makes a long move to the side of the screen, whereas in a short time the arm makes a small movement.

On the other hand, Bordoloi et al. [46] developed a 2D maze game with a BCI based on imagined movement. The imagination of the movement of both hands simultaneously moves the character up, as the imagination of the movement to close the hand to fist moves the character down, and imagination of one hand moves the character in that direction, i.e. left or right. The game is different from most BCI games, because it switches between capture state, where the BCI tries to classify the thought of the player in one of the imaginations of movement, and the pause state, where no capture is made and the player can relax. To indicate its status, the game presents the symbol of a lamp: when off, the player can relax, and when on, the player must imagine one of the control movements of the game (the lamp symbol was chosen to represent an "idea/thought"). The maze is represented by a square grid, where the square on which the player is standing, is painted in the yellow color.

Hasan and Gan [14] developed a BCI where the game command is not reached from time to time, but when the user decides so. The game is Hangman (Fig. 4a), in which the user must find out what is the "hidden" word, by testing whether different letters are part of the word. This game's BCI is based on two imagined movements, one changes the letters in selection, and other chooses that letter to be tested. The user selects two of three imagined movements detected by the game, i.e. the right hand, left hand, or feet. If the player is tired, the game will notice the performance decrease, and will adapt the control to be more permissive and identify weaker signals of choice. Lopetegui et al. [32] developed BCI Tennis (Fig. $4 \mathrm{~b}$ ), a tennis game with a gameplay similar to the classic Pong, but with a theme of the tennis game. An imagined movement of the hand/arm up moves the character upward, and down, moves the character down. The game is intended for users with physical disabilities who cannot play games with the conventional interface via mouse/keyboard.

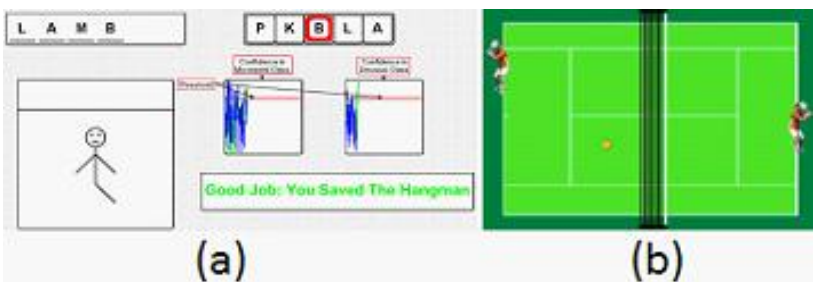

Fig. 4. Graphical interfaces of BCI games based on imagined movement (a) Hangman (b) BCI Tennis. Sources: [14][32].

Laar et al. [41] developed a relatively simple game, called BrainBasher, where the player is presented with a symbol representing the left or right hand, and then should quickly imagine the movement of that hand. Speed is the main challenge of the game, following a style of play based on reflection, but this time based purely on a BCI.

Pineda et al. [21] developed a BCI based on imagined movement for a game of the genre First Person Shooter (FPS). The BCI is used only to rotate the character clockwise or counterclockwise. The other commands are made using the conventional keyboard, for example the ' $\mathrm{S}$ ' key moves the character forward and the ' $\mathrm{x}$ ' key moves the character backwards. The time needed for training, so that the user can precisely control the character game via the $\mathrm{BCI}$ is, on average, of six and a half hours. According to the authors, the immersion in the game accelerated the training process. McCreadie et al. [28], thinking about game accessibility for visually impaired, developed Auditory Asteroids, a game where the only feedback is sonorous, i.e. no visual interface. In the pilot study, the authors develop a sound version of the game Asteroids, in which the user controls a spaceship and must avoid asteroids. Seven speakers are arranged on a sphere geometry. The control is a $\mathrm{BCI}$ based on imagined movement, with the imagination of the movement of the left and right arm, moving the ship in the corresponding direction.

Wei [31] used the BCI2000 ${ }^{6}$ system as control to a game where the player must drive a car and avoid obstacles imagining the movement of the right or left arm. The game does not punish the player drastically, in an attempt of not to discourage the user because of the difficulty found in this new interaction way: if the player hits an obstacle, the vehicle simply stops and makes a slow detour around the obstacle. Thus, the feedback error is not frustrating to the user. On the other side, Coyle et al. [45] developed a game of the asteroids genre, in which the player must avoid asteroids imagining left/right hand movement, and feet, causing the ship to move down, and tongue, causing the ship to move up. In the game, a lot of graphic elements appear to distract the player, like stars, that move across the screen. It is not needed to avoid these

\footnotetext{
${ }^{6}$ http://www.bci2000.org.
} 
additional elements, since they exist only to increase the difficulty of the game and make it more interesting.

Bonet et al. [29] study the interaction involved in a game called BrainArena, controlled by a multi-user BCI. In BrainArena the user must move a ball to right or left, imagining the movement of the hand in the same direction until the ball reaches the bottom of the screen. The authors argue that a BCI may be multi-user at four different levels: (i) level of signal processing, where signals from users are mixed in a single analysis; (ii) level of decision, in which the analysis of each user are used together, for example, finding a dominant emotion of a cine audience; (iii) the level of interaction technique, in which the users' analysis are used to perform a multi-user command, for example, for a user to drag a window, one controls direction and another controls the way, that are combined to perform a single command; and (iv) application level, where each user has independent interaction, such as control of several characters in a virtual world, each being controlled by a different user. The game developed by these authors is therefore multi-user at the decision level.

Games with some physical component, different from those purely digital, can also have an interaction by BCI. One of these works is LaFleur et al. [34], that use a BCI in a game called AR.Drone, where the player controls an Unmanned Aerial Vehicle (UAV) - a quadcopter - together with the feedback from a camera attached to the front of the UAV. In the BCI, the player must learn to imagine four hand movements: (i) the movement of the right hand turns the quadcopter clockwise; (ii) the left rotates counter-clockwise; (iii) the imagination of both hands to same time increases the height of the quadcopter; and (iv) at rest/relaxing - without imagination of hand movements - the quadcopter loses altitude. The UAV is constantly moving forward. The goal is to cross $2.30 \mathrm{~m}$ diameter rings. First the user must go through several training sessions where should learn to control the vehicle in one dimension - just rotate clockwise/anti-clockwise - to then train control in two dimensions, adding the command to raise/lower altitude with imagination of both hands simultaneously versus rest/relaxing state. Then the user must train in a virtual world, to finally control the quadcopter in a real environment. The authors use a school gym to the real environment, with two rings placed close to the center, facing one another at a distance of $5.2 \mathrm{~m}$ from each other.

Another work that embraces physical elements is $B C I$ Pinball, by Tangermann et al. [42]. These authors change the design of a conventional pinball machine, so that it is played by a BCI. The control is made through the imagined movement of the hands, where each of these movements activates one of the two pinball flippers. To adapt to the current reality of BCIs, three modifications were made: (i) the side exits were closed, so the only way out of the ball is the gap between the flippers; (ii) an obstacle was added between and slightly above the flippers, so that the ball always tends to one of the two sides since a perfectly vertical fall would require the simultaneous activation of the two flippers, which requires a very high temporal accuracy of the $\mathrm{BCI}$; and (iii) the slope of the machine was reduced to slow the ball drop. With this new machine design, BCI served as a good control, achieving even the need for high-speed response inherent to pinball games.

\section{BCI Games based on Hybrid Detection}

In the game Bacteria Hunt (Fig. 5a), developed by Mühl et al. [25], the user controls an amoeba to eat bacteria in a certain time limit. The game has four stages, each one using a different detection approach. In the first stage, control is via keyboard, but has a chance of not working. In the second stage, the concentration is used, where high levels decrease the chance of the keyboard to fail. In the third stage, SSVEP stimulus is added, when the character comes close to a bacterium, the user must focus on a circle which flashes upon the amoeba and bacteria, otherwise the bacteria escapes. In the last stage, P300 stimulus is used, the bacteria are divided into groups and each group increases in size and flashes, at a time.

Pires et al. [40] developed the BCI-Tetris (Fig. 5b), a version of Tetris game controlled by a BCI. In the game, the piece rotation is selected by $\mathrm{P} 300$ in four possible rotations, while its positioning is done by imagined movement, with the imagination of movement of the left/right hand moving the piece for the respective sides. The authors also experimented alternative designs, before deciding for the hybrid version. In a previous version, the player selected the piece and its rotation at the same time via a P300 interface. The large number of possibilities (16) decreased the accuracy, so the authors attempted a second version where the piece is automatically selected, then the player selects the rotation through P300 and finally chooses one of the four positions for the piece, also in the P300. With the addition of detection by imagined movement for the selection of the position, according to the authors, the game managed to achieve good accuracy while keeping a certain fidelity to the style of the original Tetris.

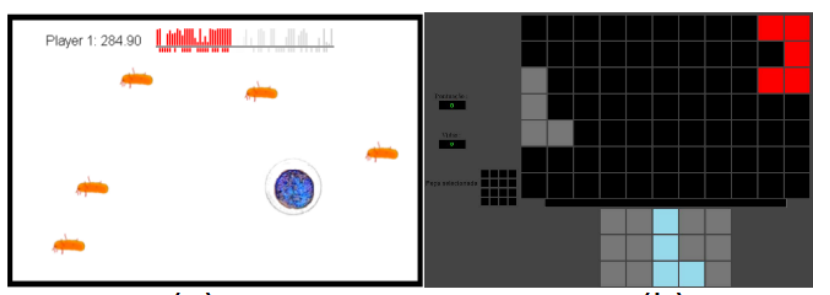

(a)

(b)

Fig. 5. Graphical interfaces of BCI games based on hibrid detection (a) Bacteria Hunt (b) BCI-Tetris. Sources: [25][40].

An adapted BCI for an existing game is presented by Maruthappan et al. [24], which developed Brain-Chess, an elaborate BCI control to play chess. The player must participate in training sessions, which also aims to capture the mental profile of the player in every step of controlling a move. The first training is done with a special memory game in which each type of chess piece is represented by a letter with a picture of a piece. In this training, it is registered a specific signature of the "thinking" associated with each piece of each user. So, after this training, it is possible to detect which piece the player wants to move. In the second part of the training, the player should imagine the movement of the chess pieces, and the signal is recorded for each type of movement, such as "move diagonally" and "move horizontally". Finally, the player selects to which square he wants the piece to move. This selection is made by P300, where the user defines the position of the board choosing a letter and a number in a matrix. 


\section{E. A Synthesis of the Main Findings of the Literature Review}

Table I presents a synthesis of the BCI-based games presented above.

TABLE I. SYNTHESIS OF THE LITERATURE BCI-BASED GAMES.

\begin{tabular}{|c|c|c|c|c|c|}
\hline \multicolumn{3}{|r|}{ Work } & \multirow{2}{*}{$\begin{array}{l}\text { Game } \\
\text { genre }\end{array}$} & \multirow{2}{*}{ Player } & \multirow{2}{*}{$\begin{array}{l}\text { Detection } \\
\text { approach }\end{array}$} \\
\hline Year & Ref. & Game name & & & \\
\hline 2009 & [9] & Finding Star & Adventure & Single & \multirow{9}{*}{ Neurofeedback } \\
\hline 2012 & {$[48]$} & (horror game) & Puzzle & Single & \\
\hline 2012 & {$[4]$} & Archery Game & Sport & Single & \\
\hline 2011 & {$[13]$} & (ADHD rehabilitation) & Sport & Single & \\
\hline 2011 & {$[7]$} & Brain Maze & Puzzle & Single & \\
\hline 2013 & {$[33]$} & Emotion Walking & Simulation & Single & \\
\hline 2012 & {$[37]$} & Lost in the Dark & Puzzle & Single & \\
\hline 2011 & [39] & Mind Garden & Simulation & Single & \\
\hline 2010 & {$[38]$} & NeuroWander & Adventure & Single & \\
\hline 2011 & {$[8]$} & \multirow{2}{*}{ Mind the Sheep } & \multirow{2}{*}{$\begin{array}{c}\text { Puzzle/ } \\
\text { Action } \\
\end{array}$} & Single & \multirow{6}{*}{$\begin{array}{c}\text { Visual Stimulus } \\
\text { (SSVEP) }\end{array}$} \\
\hline 2012 & {$[22]$} & & & Multi & \\
\hline 2004 & {$[12]$} & MindBalance & Sport & Single & \\
\hline 2007 & {$[44]$} & (2D race game) & Sport & Single & \\
\hline 2012 & {$[6]$} & (BCI tower defense) & Strategy & Single & \\
\hline 2012 & {$[23]$} & The Maze & Puzzle & Single & \\
\hline 2012 & {$[20]$} & Connect Four & Strategy & 2 players & \multirow{4}{*}{$\begin{array}{c}\text { Visual Stimulus } \\
\text { (P300) }\end{array}$} \\
\hline 2011 & {$[1]$} & Brain Invaders & Shooter & Single & \\
\hline 2012 & {$[5]$} & (memory game) & Puzzle & Single & \\
\hline 2009 & {$[43]$} & MindGame & Puzzle & Single & \\
\hline 2011 & {$[2]$} & \multirow{2}{*}{ World of Warcraft } & \multirow{2}{*}{ MMORPG } & \multirow{2}{*}{$\begin{array}{c}\text { Massive } \\
\text { multiplayer }\end{array}$} & \multirow{15}{*}{$\begin{array}{l}\text { Imagined } \\
\text { Movement }\end{array}$} \\
\hline 2012 & {$[3]$} & & & & \\
\hline 2013 & {$[16]$} & Thinking Penguin & Racing & Single & \\
\hline 2013 & {$[47]$} & (Motor rehabilitation) & Sport & Single & \\
\hline 2012 & {$[46]$} & (movement maze game) & Puzzle & Single & \\
\hline 2012 & {$[14]$} & Hangman & Puzzle & Single & \\
\hline 2011 & {$[32]$} & BCI Tennis & Sport & Multi & \\
\hline 2009 & {$[41]$} & BrainBasher & Action & Single & \\
\hline 2003 & {$[21]$} & (BCI for a FPS game) & FPS & Online multi & \\
\hline 2012 & {$[28]$} & Auditory Asteroids & Shooter & Single & \\
\hline 2010 & {$[31]$} & (cehicle control game) & Simulation & Single & \\
\hline 2011 & {$[45]$} & (asteroids-like game) & Shooter & Single & \\
\hline 2013 & {$[34]$} & (AR.Drone BCI) & Action & Single & \\
\hline 2013 & {$[29]$} & BrainArena & Sport & 2 players & \\
\hline 2008 & {$[42]$} & BCI-Pinball & Action & Single & \\
\hline 2010 & {$[25]$} & Bacteria Hunt & Action & Single & \multirow{3}{*}{$\begin{array}{c}\text { Hybrid } \\
\text { Detection }\end{array}$} \\
\hline 2011 & {$[40]$} & BCI-Tetris & Puzzle & Single & \\
\hline 2011 & {$[24]$} & Brain-Chess & Strategy & 2 players & \\
\hline
\end{tabular}

Based on the analysis of games of the literature, it is found that the first trend is the fact that games developed with a focus on BCI are usually simplified so that the BCI is able to exercise total control over it. Although, it is possible to seek the most similar/close genre to a game based on BCI, in order to find the most appropriate BCI approach. These games follow genres based on classic games, like Asteroids (shooter), Pac-Man (puzzle-maze) "snake" game (action-snake) and chess (strategy-board). This trend is due to two reasons, i.e., to simplify the necessary controls and the development of the game, so that the focus is on the BCI design.

When using an interaction based on visual stimulus, is important to take into account the pace of the game: an oscillatory stimulus, in which everything flashes simultaneously, indicates a frenetic pace and high frequencies could indicate a more dangerous element; whereas a transient stimulus, where elements flash one at a time, indicates a milder and calculated pace. As an example, a game with "bombs" could use oscillating stimulus, with higher frequencies indicating bombs about to explode, while a game of puzzle could use transient stimulus, where the user thinks quietly about the next step. Even in games based on visual stimulus, the matrix disposal usually provides greater accuracy. A matrix disposal is good for board games, in which the "world" consists of a grid of squares. Games like Minesweeper and Battleship have a direct mapping to this graphic disposal.

In turn, detection based on imagined movement appears to be most advantageous for a $3 \mathrm{D}$ environment control, and has been used in 3D shooting games (FPS), racing, and Massively Multiplayer Online (MMO). Another interesting question is the influence of social elements in these games based on BCI, as the break of the controlled environment due to social interactions outside to Mind the Sheep, the use of Connect Four in competitiveness, and multi-user interactions in the design of BrainArena. The disadvantages of certain approaches, such as limited control offered by detecting the concentration of the user, are not criteria for elimination, but for option - presenting the reality that it is difficult to develop a game that uses only the concentration, but on the other hand, to use it as part of the control of the game can be very interesting.

In Finding Star, concentration serves as a relaxed state of the character, which thus can sleep; while in WoWAlpha, concentration is used as the "anger" of the user, making the character to be transformed into its wild form. In both games, the concentration is used as part of control, managing the current difficulties of this approach.

In action games, sports, FPS and Real-Time Strategy (RTS) the biggest challenge is the time present in the gameplay. These games need reflexes and quick actions by the user, and the quick and instant pace characterized by these genres is hardly captured by current BCI. In the case of games where there is no notion of time, like puzzle games, strategy and Role-Playing Games (RPGs) based in turns, it is easier to use BCI as control. The smoother pace also resembles the high reasoning required in each step. Shooter genre games, such as asteroids or alien invaders, combine with BCIs based on visual stimulus, because enemies can be used as a focus target.

\section{DESIGN RATIONALE}

Digital games, including BCI-based games, are very diverse applications, ranging in genre, style and target audience. Therefore, it is difficult to define unique guidelines that cover all genres of digital games. A more flexible approach is the development of a Design Rationale - a reflection and documentation about the design decisions, as well as solutions and available technologies [49]. The design rationale of BCI-based games can be useful for the academic community and industry, since it outlines the major design issues that should be considered when developing games that use this new interaction way. In the design rationale, every choice is balanced with pro and con arguments, so that game designers - not necessarily specialize in BCI - can get relevant information about the design. Fig. 6 presents the design rationale developed and mapped with Compendium tool [50][51]. It is important to highlight that the development of this design rationale is based on previous results of our 
research in the context of BCI [18] and by the analysis of the BCI games from the literature, as discussed earlier.

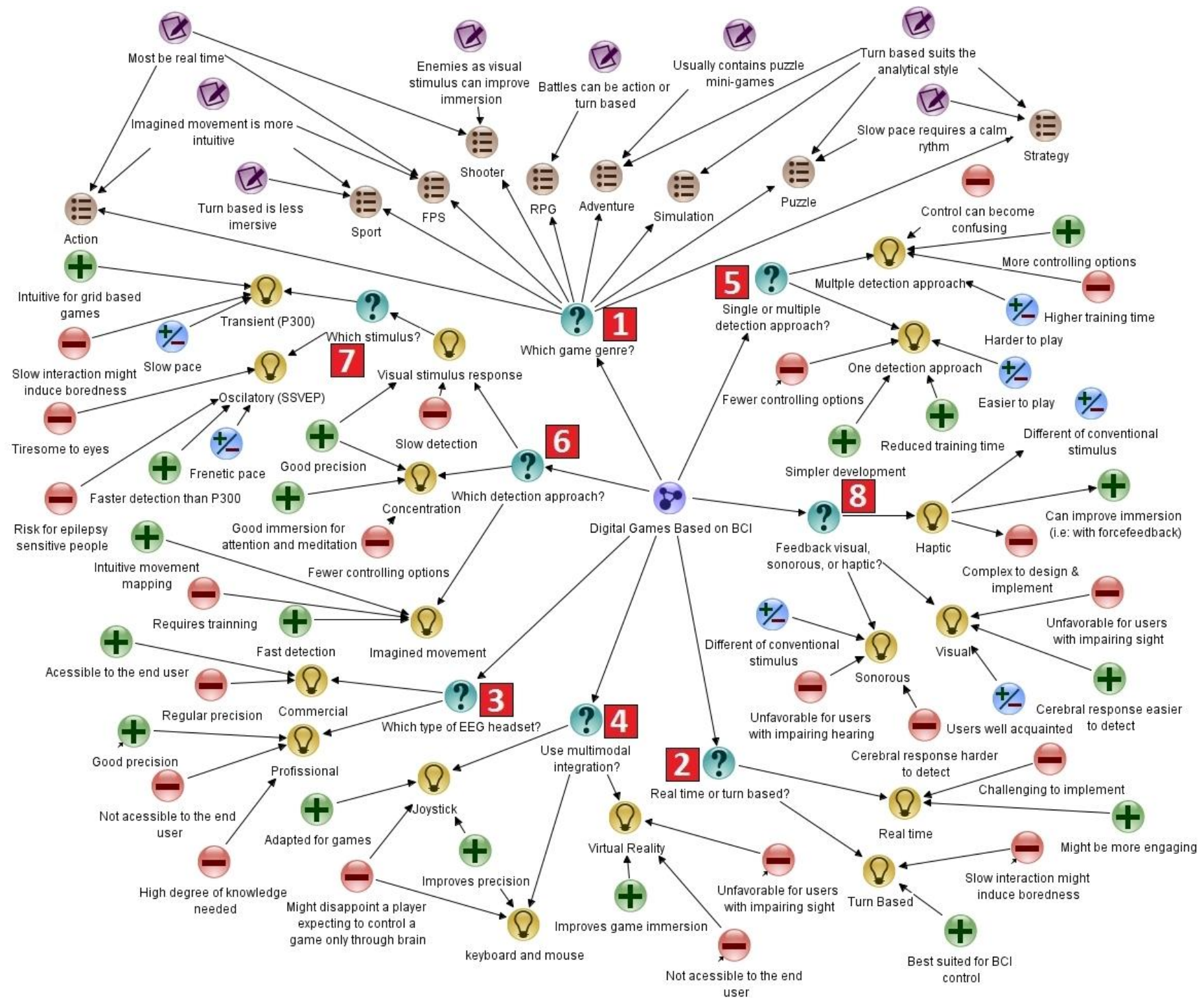

Fig. 6. Design rationale for digital games based on BCI, proposed in the present work.

The reading of the design rationale starts in "Digital games based on BCI" (represented by the icon in Fig. 7a) from where the questions/reflections start, represented by the symbol of Fig. 7b. There is no need to follow the questions in a specific order, but the design rationale suggests an order by numbering each of the questions from the first to the eighth. The seventh question has only importance in cases where detection based on visual stimulus is used, thus, it is a subquestion that comes, situationally, from question 6). Thinking first about the game genre facilitates the reflections made by other questions. Each question is linked to choices, possible answers to the question, represented by the symbol of Fig. 7c. For each of these choices, there are positive points (represented by the icon in Fig. 7d), negative points (represented by the icon in Fig. 7e), or variable, which can be pro or con depending on the context of the game design (represented by the icon in Fig. 7f). The choices of the genre questioning are represented by a different symbol (Fig. $7 \mathrm{~g}$ ) because they are a definition that comes from the concept of the game, so instead of positive/negative points, they have recommendations linked to them (represented by the symbol in Fig. 7h).

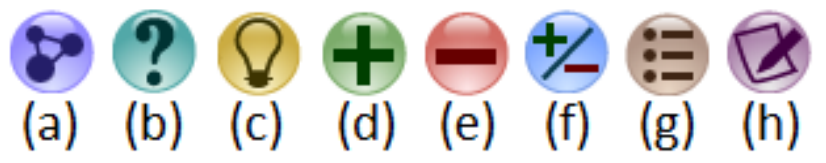

Fig. 7. Symbols used in the design rationale.

The design rationale is a tool that allows the game designer to incorporate controls via $\mathrm{BCI}$ in a game design, and to consider the current reality of this kind of interface. Hence, the designer can develop a design even without knowledge of the BCI field. Some choices may appear to have very limiting negative points, as "few control options", but in fact, they not 
affect the design of certain games, like the acclaimed game Jetpack Joyride, which has only a single button to control. Similarly, there are positive points that will not matter for a specific design. That is, the design rationale does not provide universal answers, but questions to be considered and adapted by the game designer.

As seen, the design rationale presented is intended to explain the opportunities and challenges of each approach, while guiding the designer in the construction of the game especially related to controls. The developed design rationale does not embrace decisions that depend of the target audience, such as user's age or experience, because it intends to be applicable to any game with BCI - independent of the user.

\section{ADMIRALMIND BATTLESHIP STUDY}

In this section, first, we present the methodology used in the study of AdmiralMind Battleship BCI game, and then present and discuss the results of the study.

\section{A. Methodology}

The design rationale presented is a tool to be used in the design cycle, shown in Fig. 8, being the second step of each iteration of the cycle. Initially, the game designer must think about the concepts of the game, i.e., its central ideas. In this first step, of game concept, do not set any specific control, but the goals of the game, and entertainment elements. In the second step, the designer gets support in the design rationale to analyze, and consider, the way a BCI control works in the intended game concept.

Based on the game gender, it is possible to see the recommendations suggested by the design rationale and take them into account, considering other decisions stated on the design. In the third step, the designer should define the reason for each of the decisions, defining the rationale of them. This definition is made through the design rationale, providing answers to each one of the questions set out by the design rationale. Finally, the last step of each iteration is the creation and reflection of the game design. At this stage, the game should already have a significant planning, with defined gameplay, controls, and goals. If the designer is not satisfied with the game, or if the designer wants to think about alternatives, additional iterations can be made in the design cycle of a game with BCI. The process proposed in this paper does not cover entirely the step 4 (game design) as it does not lead to a "complete" game design, but allows the designer to get closer to this goal. The design rationale does not define, for example, a graphical interface for each game.

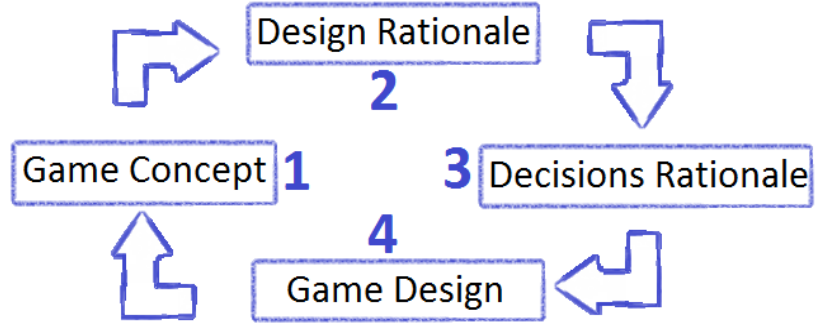

Fig. 8. Overview of the design process for digital games based on BCI, proposed in the present work.

As shown in Fig. 8, the output of each step serves as input to the next step. So, it is only possible to use the design rationale after an initial concept of the game; the setting of the decisions should be made after reading, analysis, and reflection of the design rationale; and the game design is built with the decisions made (and the reflections coming from the entire cycle of design).

\section{B. Results}

As a study of the application of the proposed design rationale, decisions are discussed for a Battleship game. The game is played by two players, and each one of them arranges their ships in the respective territory, i.e., a board in grid/matrix style. Then, they alternate in turns, choosing a square of the enemy's territory to attack. The target player indicates whether or not the shot hit, showing if there is a ship in that point. The first decision of the designer is to define the genre of the game, in this case it was decided to draw the game as "strategy", based on how battleship is played. Then, the designer thinks about the pace of the game, if it should be in real time or turn based. It is decided to be turn based, based again in the original game.

Now the designer must make several decisions about the BCI game. What headset use, commercial or professional? Using design rationale, the designer observes that professionals have better accuracy, but it is difficult to acquire - usually present only in research or medical centers. On the other hand, commercial headsets are easier to get and to use. Commercial headsets have less precision, but reach a larger audience. Thus, the designer decides to use a commercial headset. To detection approach, it is desired one that offers enough control to select multiple squares on the board, so by eliminating the concentration way (limited control), and favoring detection by imagined movement - that would be used the imagined movement of the hand to navigate the board, and of the feet to select - and the one based on visual stimulus - where the visual focus allows the selection of the desired square. The imagined movement approach requires training, but it offers a faster interaction and does not depend on game's graphic elements; whilst the visual stimulus approach does not need training, but it has a slow interaction and depends on arbitrary changes on the game visual interface - as continuous flashes. If the visual stimulus is used, the P300 approach seems more appropriate, as it has a good mapping to grid, and mild pace stimulates strategic thinking. 
The SSVEP approach seems to be unfavorable, the large amount of square flashing intermittently would cause great discomfort to the user. Then, it is decided what kind of headset to use, professional or commercial? It is expected that the game is played by people outside the academic world, so the best way would be to use a commercial headset - accessible to the target audience. As the game is simple - and has few controls a multimodal complement with keyboard and mouse would make the game less interesting, and a form of hybrid detection would be confusing and unnecessary, so it is best that the game is purely controlled by a BCI, and only one detection approach. Still about multimodal interaction, a virtual reality environment is attractive, but the high cost of the equipment would not be accessible to the end user, and would project more complex requiring a more elaborate graphical interface. Finally, it is defined that the feedback should have be mainly visual, but also a sonorous feedback, indicating right or wrong, would enrich the user experience.

TABLE II. RESULTS OF THE DECISIONS RATIONALE FOR A BCI GAME.

\begin{tabular}{|c|c|c|}
\hline $\begin{array}{c}\text { Design } \\
\text { decision }\end{array}$ & Rationale & Game design \\
\hline $\begin{array}{l}\text { 1. What is the } \\
\text { game genre? }\end{array}$ & Strategy. & $\begin{array}{l}\text { Guessing Game, as the pen } \\
\text { and paper game. }\end{array}$ \\
\hline $\begin{array}{l}\text { 2. Real time or } \\
\text { turn based? }\end{array}$ & $\begin{array}{l}\text { The design will follow the } \\
\text { original style of the game, in } \\
\text { which the players alternate } \\
\text { in turns. }\end{array}$ & $\begin{array}{l}\text { Each player must choose an } \\
\text { opponent's board position, } \\
\text { which indicates the hit or } \\
\text { miss - ending the turn. }\end{array}$ \\
\hline $\begin{array}{l}\text { 3. Commercial } \\
\text { or professional } \\
\text { headset? }\end{array}$ & $\begin{array}{l}\text { Commercial, once the target } \\
\text { user are gamers with lower } \\
\text { purchasing power. }\end{array}$ & $\begin{array}{l}\text { The headset will be EPOC, } \\
\text { which has a good cost- } \\
\text { benefit ratio between ease of } \\
\text { acquisition and resources. }\end{array}$ \\
\hline $\begin{array}{l}\text { 4. Do use of } \\
\text { multimodal } \\
\text { interface? }\end{array}$ & $\begin{array}{l}\text { No. Battleship has limited } \\
\text { commands, the player just } \\
\text { chooses a square by turn. } \\
\text { More entries would make } \\
\text { the game more complex } \\
\text { without offering benefits in } \\
\text { return. }\end{array}$ & $\begin{array}{l}\text { The game is controlled only } \\
\text { by BCI. }\end{array}$ \\
\hline $\begin{array}{l}\text { 5. One } \\
\text { detection } \\
\text { approach or } \\
\text { hybrid } \\
\text { detection? }\end{array}$ & $\begin{array}{l}\text { Hybrid approach. A hybrid } \\
\text { approach can be more } \\
\text { flexible to the player if used } \\
\text { with wisdom, despite being } \\
\text { necessary to be cautious to } \\
\text { avoid a confusing control. }\end{array}$ & $\begin{array}{l}\text { The game will provide more } \\
\text { than one detection approach, } \\
\text { but only one is active at a } \\
\text { time. This will allow the } \\
\text { player to select the preferred } \\
\text { interaction. }\end{array}$ \\
\hline $\begin{array}{l}\text { 6. Which } \\
\text { detection } \\
\text { approach to be } \\
\text { used? }\end{array}$ & $\begin{array}{l}\text { Visual stimulus, since the } \\
\text { game environment is a grid } \\
\text { - which is the classic matrix } \\
\text { interface. } \\
\text { flashes gurthermore, } \\
\text { impression of a battlefield. } \\
\text { Additionally, it will be used } \\
\text { detection based on imagined } \\
\text { movement - simulating the } \\
\text { movement, in the real game, } \\
\text { of moving the pin to the } \\
\text { target. }\end{array}$ & $\begin{array}{l}\text { The opponent's board } \\
\text { squares will act as visual } \\
\text { stimuli, flashing at the time } \\
\text { of selection. In the imagined } \\
\text { movement mode, the player } \\
\text { will be able to control a } \\
\text { cursor by moving left/right } \\
\text { with the imagination of the } \\
\text { movement of the arms, } \\
\text { moving down with the feet, } \\
\text { and moving up with the } \\
\text { imagination of arms' } \\
\text { movement together } \\
\text { simultaneously. }\end{array}$ \\
\hline $\begin{array}{l}\text { 7. Which } \\
\text { stimulus to be } \\
\text { used? }\end{array}$ & $\begin{array}{l}\text { P300, since a milder pace } \\
\text { stimulates thinking and } \\
\text { turn's strategy. }\end{array}$ & $\begin{array}{l}\text { The rows and columns will } \\
\text { flash randomly, causing an } \\
\text { impression of danger. }\end{array}$ \\
\hline $\begin{array}{l}\text { 8. Feedback } \\
\text { visual, } \\
\text { sonorous or } \\
\text { haptic? }\end{array}$ & $\begin{array}{l}\text { Visual, considering that the } \\
\text { game depends on visual } \\
\text { elements because of the BCI } \\
\text { detection approach chosen. } \\
\text { Additionally, an audio }\end{array}$ & $\begin{array}{l}\text { The constant feedback from } \\
\text { the game will be visual, but } \\
\text { there will be audio tips to } \\
\text { indicate the player's turn, } \\
\text { and a blast to indicate a hit. }\end{array}$ \\
\hline
\end{tabular}

\begin{tabular}{|l|l|l|}
\hline $\begin{array}{c}\text { Design } \\
\text { decision }\end{array}$ & \multicolumn{1}{|c|}{ Rationale } & Game design \\
\hline & $\begin{array}{l}\text { feedback is necessary to } \\
\text { indicate hit or miss of the } \\
\text { target. }\end{array}$ & \\
\hline
\end{tabular}

It is important to detach that the design rationale outlines the questions to be answered by designing a game based on $\mathrm{BCI}$, but does not define specific responses such as, for example, related to the graphical interface elements of the game. The designer can choose the alternatives, being aware of the pro and con of each one, and adapting it to reality, finally applying the design rationale for the specific scenario. The presentation of the battleship game scenario, as described above, illustrated how the design rationale developed in this work can help in the reflection of the designer, regarding the design decisions of a digital game based on BCI.

\section{Discussion}

Digital games based on BCI have a different interaction reality, compared to games with conventional controls, e.g. via keyboard and mouse. The BCI control has great potential for immersion, and can be used in various game genres. Several of the literature works show solutions with multimodal interaction, i.e. BCI complemented with another interaction way. This demonstrates, in a way, how complex is the game design based only on BCI.

The use of BCIs in games causes a strong impact on the interaction design, changing gameplay aspects, even related to the graphical interface. Neurofeedback based games often use a bar to indicate the level of attention/relaxation of the user (e.g. both in Fig. 1). On the other hand, games based on visual stimulus need elements that are strange to the game (that operate as the stimuli). In Mind the Sheep, the three flashing circles clashes from the scenario (Fig. 2a), while in MindBalance game, it was necessary to insert checkered flags on each side of character's rod, notwithstanding balance rods have no flags (Fig. 2b). Still, in The Maze, it was necessary to insert huge arrows, occupying half of the screen; arrows that would not exist if this game did not use BCI (Fig. 3a). In Connect Four, columns become out of visibility, hidden by the swinging bar, which complicates the player's reasoning (Fig. $3 b)$.

On the other hand, some games based on BCI add unnecessary elements such as a graphic with the reading of brain waves $-\mathrm{a}$ feedback difficult to be understood by a player (Fig. 4a/Fig. 5a). The care with the graphical interface is more important in games with hybrid detection, because in these games, control is already complicated and it is difficult to deduce how to play. Choices - such as detection approach will impact on gameplay and graphical interface. However, the purpose of the design rationale is not to define the graphical interface of the game, but rather support the designer, bringing reflections of how to use $\mathrm{BCI}$ in a game.

In games with the "stealth" element, the user should be relaxed to improve the ability to keep on hidden; in puzzle games, mental effort combines with the fun; and in games with virtual reality, BCI extends the feeling of being in another "world". It is understood that the design rationale, as described, 
supports the designer in the design of new BCI-based games. It is difficult to adapt conventional games for BCI, as they were developed and guided by other requirements. The pace in conventional games can be very fast, and the required accuracy is very high. Whereas, in the case of BCI, it is necessary to adapt to the current reality, where EEG detection has a delay and consists of an interaction with a slow pace.

Most of these BCI controls require a lot of concentration/focus from the user, which can be complicated in situations of actual use, as the work of Obbink et al. [22] shows, in which the user is not able to "pause" the BCI control. In the literature, most BCI games are based on visual stimulus, generating an accessibility problem that complicates or forbids its use by visually impaired users. Moreover, it affects cohesion/immersion of the design, since having many stimuli like flashing circles in black and white - clash of the visual style of the game, and visual elements become forced and artificial. A good adaptation can minimize, or even eliminate, these challenges of BCI games. Then, the design rationale helps in creating a game whose BCI control is well adapted, improving the user experience. Another benefit of the design rationale is to encapsulate the knowledge about the BCI field, allowing the design of games by developers who have low knowledge in BCI.

BCIs change significantly the interaction way with systems in the area of BCI games, bringing new interaction ways that create a new connection to the player, such as: the creation of virtual "members" through imagined movement; the transfer of the state of mind to the game characters - which relax or concentrate along with the player; the mental selection with the visual image currently in the brain (the one being focused); the brain control of objects (like the telekinetic power of the gaming world); and even a link between player's heart with the game character's - in which emotions like fear are explored in the game. This provides great immersion potential of the game: the player must remain calm as the secret agent infiltrated in the enemy base; the player can feel the fear of the hero in the sudden appearance of a spirit; and imagining an arm wielding a sword, the player can hit a heroic stroke to the villain who terrorizes the city.

This work deals with any type of game controlled by BCI, without being attached to a specific genre or approach. But at the same time, it identifies the differences in each game, arguing that the design depends on the scenario and game desired. Still, it does not point out strict rules to be followed, but the decisions to be made by the designer during the design of a BCI-based game. There are not ideal approaches, it is necessary to adapt to the context and take into consideration that each one has pro and con points. This work presents, as far as we know, the first design rationale to guide designers in the design of new BCI-based digital games. The design rationale is able to cover all kinds of games, without losing the specificity required for each case. Hence, it contributes to any designer interested in developing a game based on BCI.

\section{CONCLUSION}

This paper presented a literature review about digital games based on BCI. Based on this survey, and on previous results of our research in this area, we developed a design rationale to support game designers in designing digital games based on BCI. Still, we conducted a study where we apply the design rationale in the design process of a naval battleship game controlled by BCI.

As future work, based on the results of this work, we suggest the implementation/coding of AdmiralMind Battleship, whose early stages of the design process were supported by the design rationale presented in this work. Later, it is still considered relevant to evaluate the interface and user experience with the target audience of this BCI game.

\section{ACKNOWLEDGMENT}

This work was partially supported by the Brazilian Federal Agency for Support and Evaluation of Graduate Education (CAPES), by the Brazilian National Council of Scientific and Technological Development (CNPq grant \#163408/2012-2), and by the Physical Artifacts of Interaction Research Group (PAIRG) at Federal University of Rio Grande do Norte (UFRN), Brazil.

\section{REFERENCES}

[1] M. Congedo, M. Goyat, N. Tarrin, G. Ionescu, L. Varnet, B. Rivet, R. Phlypo, N. Jrad, M. Acquadro, and C. Jutten, “'Brain Invaders': a prototype of an open-source P300-based video game working with the OpenViBE platform," in Proceedings of the $5^{\text {th }}$ International BrainComputer Interface Conference (BCI'11), HAL, 2011, pp. 280-283.

[2] R. Scherer, E. Friedrich, B. Allison, M. Pröll, M. Chung, W. Cheung, R. Rao, and C. Neuper, "Non-invasive brain-computer interfaces: enhanced gaming and robotic control," in Proceedings of the $11^{\text {th }}$ International Work-Conference on Artificial Neural Networks (IWANN'11), Springer, 2011, pp. 362-369, doi: 10.1007/978-3-642-21501-8_45.

[3] R. Scherer, M. Proll, B. Allison, and G.R. Muller-Putz, "New input modalities for modern game design and virtual embodiment," in Proceedings of the IEEE Virtual Reality Short Papers and Posters (VRW'12), IEEE, 2012, pp. 163-164, doi: 10.1109/VR.2012.6180932.

[4] L.-D. Liao, C.-Y. Chen, I.J. Wang, S.-F. Chen, S.-Y. Li, B.-W. Chen, J.Y. Chang, and C.-T. Lin, "Gaming control using a wearable and wireless EEG-based brain-computer interface device with novel dry foam-based sensors," in Journal of Neuroengineering and Rehabilitation, vol. 9, no. 5, BioMed Central, 2012, pp. $1-12$, doi: 10.1186/1743-0003-9-5.

[5] C. Angeloni, D. Salter, V. Corbit, T. Lorence, Y.-C. Yu, and L.A. Gabel, "P300-based brain-computer interface memory game to improve motivation and performance," in Proceedings of the $38^{\text {th }}$ Annual Northeast Bioengineering Conference (NEBEC'12), IEEE, 2012, pp. 35-36, doi: 10.1109/NEBC.2012.6206949.

[6] V.M. Vliet, A. Robben, N. Chumerin, N.V. Manyakov, A. Combaz, and M.M. Van Hulle, "Designing a brain-computer interface controlled video-game using consumer grade EEG hardware," in Proceedings of the ISSNIP Biosignals and Biorobotics Conference (BRC'12), IEEE, 2012, pp. 1-6, doi: 10.1109/BRC.2012.6222186.

[7] P. Coulton, C.G. Wylie, and W. Bamford, "Brain interaction for mobile games," in Proceedings of the $15^{\text {th }}$ International Academic MindTrek Conference (MindTrek'11), ACM, 2011, pp. 37-44, doi: 10.1145/2181037.2181045.

[8] G. Hakvoort, H. Gürkök, D.P. Bos, M. Obbink, and M. Poel, "Measuring immersion and affect in a brain-computer interface game," in Proceedings of the $13^{\text {th }}$ IFIP TC 13 International Conference on Human-Computer Interaction (INTERACT'11), Springer, 2011, pp. 115-128, doi: 10.1007/978-3-642-23774-4_12.

[9] M. Ko, K. Bae, G. Oh, and T. Ryu, "A study on new gameplay based on brain-computer interface,"in Proceedings of the Digital Games Research Association (DiGRA’09), Brunel University, 2009, pp. 1-7. 
[10] F. Lotte, "Brain-computer interfaces for 3D games: hype or hope?," in Proceedings of the $6^{\text {th }}$ International Conference on Foundations of Digital Games (FDG'11), ACM, 2011, pp. 325-327, doi: $10.1145 / 2159365.2159427$.

[11] H. Gürkök, A. Nijholt, and M. Poel, "Brain-computer interface games: towards a framework," in Proceedings of the $11^{\text {th }}$ International Conference on Entertainment Computing (ICEC'12), Springer, 2012, pp. 373-380, doi: 10.1007/978-3-642-33542-6_33.

[12] E. Lalor, S.P. Kelly, C. Finucane, R. Burke, R.B. Reilly, and G. McDarby, "Brain computer interface based on the steady-state VEP for immersive gaming control," in Biomedizinsche Tecknik, vol. 49, no. 1, 2004, pp. 63-64.

[13] L. Jiang, C. Guan, H. Zhang, C. Wang, and B. Jiang, "Brain computer interface based 3D game for attention training and rehabilitation," in Proceedings of the $6^{\text {th }}$ IEEE Conference on Industrial Electronics and Applications (ICIEA'11), IEEE, 2011, pp. 124-127, doi: 10.1109/ICIEA.2011.5975562.

[14] B.A.S. Hasan and J.Q. Gan, "Hangman BCI: an unsupervised adaptive self-paced brain-computer interface for playing games," in Computers in Biology and Medicine, vol. 42, no. 5, Elsevier, 2012, pp. 598-606, doi: 10.1016/j.compbiomed.2012.02.004.

[15] Y. Sung, K. Cho, and K. Um, "A development architecture for serious games using BCI (brain computer interface) sensors," in Sensors, vol. 12, no. 11, MDPI, 2012, pp. 15671-15688, doi: 10.3390/s121115671.

[16] R. Leeb, M. Lancelle, V. Kaiser, D.W. Fellner, and G. Pfurtscheller, "Thinking Penguin: multimodal brain-computer interface control of a VR game," in IEEE Transactions on Computational Intelligence and AI in Games, vol. 5, no. 2, IEEE, 2013, pp. 117-128, doi: 10.1109/TCIAIG.2013.2242072.

[17] A. Kaplan, S. Shishkin, I. Ganin, I. Basyul, and A. Zhigalov, "Adapting the P300-based brain-computer interface for gaming: a review," in IEEE Transactions on Computational Intelligence and AI in Games, vol. 5, no. 2, IEEE, 2013, pp. 141-149, doi: 10.1109/TCIAIG.2012.2237517.

[18] Ferreira, A.L.S., Miranda, L.C., Miranda, E.E.C., and Sakamoto, S.G., "A survey of interactive systems based on brain-computer interfaces," in SBC Journal on 3D Interactive Systems, vol. 4, no. 1, SBC, 2013, pp. 313.

[19] G.F.P. van Veen, "Thought games: advancements in gaming with a brain-computer interface from a game design perspective," Internet: http://hmi.ewi.utwente.nl/verslagen/capita-selecta/RT-Veen-Gijsvan.pdf [Aug. 31, 2013].

[20] E. Maby, M. Perrin, O. Bertrand, G. Sanchez, and J. Mattout, "BCI could make old two-player games even more fun: a proof of concept with 'Connect Four',' in Advances in Human-Computer Interaction, vol. 2012, 2012, pp. 1-8, doi: 10.1155/2012/124728.

[21] J.A. Pineda, D.S. Silverman, A. Vankov, and J. Hestenes, "Learning to control brain rhythms: making a brain-computer interface possible," in IEEE Transactions on Neural Systems and Rehabilitation Engineering, vol. 11, no. 2, IEEE, 2003, pp. 181-184, doi: 10.1109/TNSRE.2003.814445.

[22] M. Obbink, H. Gürkök, D.P.O. Bos, G. Hakvoort, M. Poel, and A. Nijholt, "Social interaction in a cooperative brain-computer interface game," in Proceedings of the $4^{\text {th }}$ International ICST Conference on Intelligent Technologies for Interactive Entertainment (INTETAIN'11), Springer, 2012, pp. 183-192, doi: 10.1007/978-3-642-30214-5_20.

[23] N. Chumerin, N.V. Manyakov, A. Combaz, A. Robben, M. van Vliet, and M.M. Van Hulle, "Steady state visual evoked potential based computer gaming - the Maze," in Proceedings of the $4^{\text {th }}$ International ICST Conference on Intelligent Technologies for Interactive Entertainment (INTETAIN'11), Springer, 2012, pp. 28-37, doi: 10.1007/978-3-642-30214-5_4.

[24] N. Maruthappan, N. Iyengar, and P.S. Patel, "Brain chess - playing chess using brain computer interface," in Proceedings of the International Conferece on Advancement in Information Technology (ICAIT'11), IPCSIT, 2011, pp. $183-191$.

[25] C. Mühl, H. Gürkök, D. Bos, M. Thurlings, L. Scherffig, M. Duvinage, A. Elbakyan, S. Kang, M. Poel, and D. Heylen, "Bacteria hunt," in Journal on Multimodal User Interfaces, vol. 4, no. 1, Springer, 2010, pp. 11-25, doi: 10.1007/s12193-010-0046-0.
[26] D. Bos, B. Reuderink, B. Laar, H. Gürkök, C. Muhl, M. Poel, D. Heylen, and A. Nijholt, "A human-computer interaction for BCI games: usability and user experience," in Proceedings of the International Conference on Cyberworlds (CW'10), IEEE, 2010, pp. 277-281, doi: 10.1109/CW.2010.22.

[27] J. Lee and K.Y. Lai, "What's in design rationale?," in Human-Computer Interaction, vol. 6, no. 3-4, Taylor \& Francis, 1991, pp. 251-280, doi: 10.1080/07370024.1991.9667169.

[28] K. McCreadie, D.H. Coyle, and G. Prasad, "Sensorimotor-rhythm modulation feedback with 3D vector-base amplitude panning - a braincomputer interface pilot study," in Proceedings of the Irish Signals and Systems Conference, IET, 2012, pp. 1-6.

[29] L. Bonnet, F. Lotte, and A. Lecuyer, "Two brains, one game: design and evaluation of a multiuser BCI video game based on motor imagery," in IEEE Transactions on Computational Intelligence and AI in Games, vol. 5, no. 2, IEEE, 2013, pp. 185-198, doi: 10.1109/TCIAIG.2012.2237173.

[30] D. Marshall, D. Coyle, S. Wilson, and M. Callaghan, "Games, gameplay, and BCI: the state of the art," in IEEE Transactions on Computational Intelligence and AI in Games, vol. 5, no. 2, IEEE, 2013, pp. 82-99, doi: 10.1109/TCIAIG.2013.2263555.

[31] P. Wei, "An virtual vehicle control game for online brain computer interface feedback training," in Proceedings of the International Conference on Mechatronics and Automation (ICMA'10), IEEE, 2010, pp. 1942-1944, doi: 10.1109/ICMA.2010.5589104.

[32] E. Lopetegui, B. Garcia Zapirain, and A. Mendez, "Tennis computer game with brain control using EEG signals," in Proceedings of the $16^{\text {th }}$ International Conference on Computer Games (CGAMES'11), IEEE, 2011, pp. 228-234, doi: 10.1109/CGAMES.2011.6000344.

[33] A.H. Basori, "Emotion walking for humanoid avatars using brain signals," in International Journal of Advanced Robotic Systems, InTech, 2013, pp. 1-11, doi: 10.5772/54764.

[34] K. LaFleur, K. Cassady, A. Doud, K. Shades, E. Rogin, and B. He, "Quadcopter control in three-dimensional space using a noninvasive motor imagery-based brain-computer interface," in Journal of Neural Engineering, vol. 10, no. 4, IOP, 2013, doi: 10.1088/17412560/10/4/046003.

[35] L.C. Miranda, H.H. Hornung, and M.C.C. Baranauskas, "Adjustable interactive rings for iDTV," in IEEE Transactions on Consumer Electronics, vol. 56, no. 3, IEEE, 2010, pp. 1988-1996, doi: 10.1109/TCE.2010.5606356.

[36] L.C. Miranda, H.H. Hornung, R. Pereira, and M.C.C. Baranauskas, "Exploring adjustable interactive rings in game playing: preliminary results," in Proceedings of the $2^{\text {nd }}$ International Conference on Design, User Experience and Usability (DUXU/HCII'13), Springer, 2013, pp. 518-527, doi: 10.1007/978-3-642-39241-2_57.

[37] R. Bernays, J. Mone, P. Yau, M. Murcia, J. Gonzalez-Sanchez, M.E. Chavez-Echeagaray, R.M. Christopherson, and R. Atkinson, "Lost in the dark: emotion adaption," in Adjunct Proceedings of the $25^{\text {th }}$ ACM Symposium on User Interface Software and Technology (UIST Adjunct'12), ACM, 2012, pp. 79-80, doi: 10.1145/2380296.2380331.

[38] M.S. Yoh, J. Kwon, and S. Kim, "NeuroWander: a BCI game in the form of interactive fairy tale," in Proceedings of the $12^{\text {th }}$ ACM International Conference Adjunct Papers on Ubiquitous Computing Adjunct (Ubicomp'10 Adjunct), ACM, 2010, pp. 389-390, doi: 10.1145/1864431.1864450.

[39] L. Su, M. Wenyan, and H. Qiqian, "The Mind Garden: a brain computer interface game," Internet: https://wiki.cc.gatech.edu/designcomp/images/2/24/MindGardenFinalRe port.pdf [Aug. 31, 2013].

[40] G. Pires, M. Torres, N. Casaleiro, U. Nunes, and M. Castelo-Branco, "Playing tetris with non-invasive BCI," in Proceedings of the $1^{\text {st }}$ IEEE International Conference on Serious Games and Applications for Health (SeGAH'11), IEEE, 2011, pp. 1-6, doi: 10.1109/SeGAH.2011.6165454.

[41] B. van de Laar, R. Boris, D.P.-O. Bos, and D. Heylen, "Evaluating user experience of actual and imagined movement in BCI gamin," in International Journal of Gaming and Computer-Mediated Simulations, vol. 2, no. 4, IGI Global, 2010, pp. 33-47, doi: 10.4018 /jgcms.2010100103. 
[42] M. Tangermann, M. Krauledat, K. Grzeska, M. Sagebaum, B. Blankertz, C. Vidaurre, and K.-R. Müller, "Playing pinball with non-invasive BCI," in Proceedings of the $22^{\text {nd }}$ Annual Conference on Neural Information Processing Systems (NIPS'08), 2008, pp. 1641-1648.

[43] A. Finke, A. Lenhardt, and H. Ritter, "The MindGame: a P300-based brain-computer interface game," in Neural Networks, vol. 22, no. 9, Elsevier, 2009, pp. 1329-1333, doi: 10.1016/j.neunet.2009.07.003.

[44] P. Martinez, H. Bakardjian, and A. Cichocki, "Fully online multicommand brain-computer interface with visual neurofeedback using SSVEP paradigm," in Computational Intelligence and Neuroscience, vol. 2007, pp. 13-13, doi: 10.1155/2007/94561.

[45] D. Coyle, J. Garcia, A.R. Satti, and T.M. McGinnity, "EEG-based continuous control of a game using a 3 channel motor imagery BCI: BCI game," in Proceedings of the IEEE Symposium on Computational Intelligence, Cognitive Algorithms, Mind, and Brain (CCMB'11), IEEE, 2011, pp. 1-7, doi: 10.1109/CCMB.2011.5952128.

[46] S. Bordoloi, U. Sharmah, and S.M. Hazarika, "Motor imagery based $\mathrm{BCI}$ for a maze game," in Proceedings of the $4^{\text {th }}$ International Conference on Intelligent Human Computer Interaction (IHCI'12), IEEE, 2012, pp. 1-6, doi: 10.1109/IHCI.2012.6481848.
[47] B. Badia, A. García Morgade, H. Samaha, and P.F.M.J. Verschure, "Using a hybrid brain computer interface and virtual reality system to monitor and promote cortical reorganization through motor activity and motor imagery training," in IEEE Transactions on Neural Systems and Rehabilitation Engineering, vol. 21, no. 2, IEEE, 2013, pp. 174-181, doi: 10.1109/TNSRE.2012.2229295.

[48] T.R. Kang, I. Perez, and G.L.E. Matias, "Design and development of an affect-sensitive horror game," in Proceedings of the $12^{\text {th }}$ Philippine Computing Science Congress, 2012.

[49] H. Hornung and M.C.C. Baranauskas, "Towards a design rationale for inclusive eGovernment services," in International Journal of Electronic Government Research, vol. 7, no. 3, IGI Global, 2011, pp. 1-20, doi: 10.4018/jegr.2011070101.

[50] S.J.B. Shum, A.M. Selvin, M. Sierhuis, J. Conklin, C.B. Haley, and B. Nuseibeh, "Hypermedia support for argumentation-based rationale," in Rationale management in software engineering, Springer, 2006, pp. 111-132, doi: 10.1007/978-3-540-30998-7_5.

[51] A. Brasher, G. Conole, S. Cross, M. Weller, P. Clark, and J. White, "CompendiumLD - a tool for effective, efficient and creative learning design," in Proceedings of the 2008 European LAMS Conference: Practical Benefits of Learning Design, 2008. 